\title{
ASPECTS OF VALUE DISTRIBUTION THEORY IN SEVERAL COMPLEX VARIABLES
}

\author{
BY WILHELM STOLL
}

During the last fifty years value distribution in one complex variable has been established as one of the most beautiful branches of complex analysis. In several variables, value distribution was slow to grow up. Only a few people were concerned and many obstacles had to be overcome. However, recently, the theory has gained wide recognition. The outlook for the future is bright and promises a theory even broader in scope than its one-dimensional counterpart.

1. The classical theory. At first let us look at some basic results in one variable. Realize the Riemann sphere $\mathbf{P}_{1}$ as a sphere of diameter 1 in $\mathbf{R}^{3}$. The chordal distance between points $w$ and $a$ in $\mathbf{P}_{1}$ is denoted by $\|w, a\|$. Then $0 \leqslant\|w, a\| \leqslant 1$. The Riemann sphere carries a rotation invariant volume element $\Omega$ giving the sphere total volume 1 . As on each complex manifold, the exterior derivative $d=\partial+\bar{\partial}$ twists to

$$
d^{c}=(i / 4 \pi)(\bar{\partial}-\partial)
$$

On $\mathbf{P}_{1}-\{a\}$, the volume element $\Omega$ is computed by

$$
\Omega=-d d^{c} \log \|a, w\|^{2} .
$$

If $r>0$, let $\mathbf{C}[r]$ be the closed disc, $\mathbf{C}(r)$ be the open disc and $\mathbf{C}\langle r\rangle$ be the circle, all of radius $r$ and with center 0 . Let $f: \mathbf{C} \rightarrow \mathbf{P}_{1}$ be a nonconstant holomorphic map, i.e., a nonconstant meromorphic function. The spherical image of $f$ is defined by

$$
A_{f}(r)=\int_{\mathbf{C}(r)} f^{*}(\Omega)>0 .
$$

For $0<s<r$, the Ahlfors-Shimizu characteristic of $f$ is defined by

$$
\left(T_{f}(r, s)\right)=\int_{s}^{r} A_{f}(t) d t / t
$$

Then $T_{f}(r, s) \rightarrow \infty$ for $r \rightarrow \infty$. On $\mathbf{C}\langle r\rangle$, a rotation invariant line element $\sigma$ exists which gives the circle $\mathbf{C}\langle r\rangle$ length 1. For $r>0$, the compensation function of $f$ for $a \in \mathbf{P}_{1}$ is defined by

$$
m_{f}(r, a)=\int_{\mathbf{C}\langle r\rangle} \log \frac{1}{\|f, a\|} \sigma \geqslant 0 .
$$

This is an expanded version of an invited address presented at the 79th Summer Meeting of the American Mathematical Society in Kalamazoo, Michigan, August 21, 1975. The author's research was partially supported by the National Science Foundation Grant MPS75-07086; received by the editors May 3, 1976.

AMS (MOS) subject classifications (1970). Primary 32H25, 32H99; Secondary $32 \mathrm{~F} 99$.

Key words and phrases. Value distribution, First Main Theorem, Second Main Theorem, Defect Relation, parabolic manifolds. 
Let $\theta_{f a}(z)$ be the $a$-multiplicity of $f$ at $z \in \mathbf{C}$. For $r>0$, the counting function

$$
n_{f}(r, a)=\sum_{z \in \mathbf{C}[r]} \theta_{f a}(z)
$$

is the number of $a$-points in $\mathbf{C}[r]$. For $0<s<r$, the valence function of $f$ to $a \in \mathbf{P}_{1}$ is defined by

$$
N_{f}(r, s ; a)=\int_{s}^{r} n_{f}(t ; a) \frac{d t}{t} .
$$

The First Main Theorem asserts

$$
T_{f}(r, s)=N_{f}(r, s ; a)+m_{f}(r, a)-m_{f}(s, a)
$$

for $0<s<r$. The Mean Value Theorem states

$$
T_{f}(r, s)=\int_{\mathbf{P}_{1}} N_{f}(r, s ; a) \Omega(a) .
$$

So the growth measure $T_{f}$ of $f$ is the average of the growth measure of the fibers of $f$. The First Main Theorem implies

$$
0 \leqslant \delta_{f}(a)=\liminf _{r \rightarrow \infty} \frac{m_{f}(r ; a)}{T_{f}(r, s)}=1-\limsup _{r \rightarrow \infty} \frac{N_{f}(r, s ; a)}{T_{f}(r, s)} \leqslant 1 .
$$

Here $\delta_{f}(a)$ is called the Nevanlinna defect of $f$ at $a$. The Mean Value Theorem implies $\int_{\mathbf{P}_{1}} \delta_{f}(a) \Omega(a)=0$. Hence $\delta_{f}(a)=0$ for almost all $a \in \mathbf{P}_{1}$. Therefore almost all fibers grow as quickly as $f$ itself. A much sharper result is Nevanlinna's Defect Relation

$$
\sum_{a \in \mathbf{P}_{1}} \delta_{f}(a) \leqslant 2
$$

Therefore $\delta_{f}(a)>0$ for at most countably many points $a \in \mathbf{P}_{1}$. Also $\delta_{f}(a)=$ 1 can occur only twice. If $f^{-1}(a)=\varnothing$, then $\delta_{f}(a)=1$. Hence the theorem of Picard that $f$ omits at most two values is obtained.

2. The general First Main Theorem in several variables. Let $M$ and $N$ be connected, complex manifolds of dimensions $m$ and $n$, respectively. Assume that a family $E=\left\{E_{a}\right\}_{a \in A}$ of analytic subsets $E_{a}$ of $N$ is given. Each $E_{a}$ has pure dimension $n-s<n$. Let $f: M \rightarrow N$ be a holomorphic map. Define $F_{a}=f^{-1}\left(E_{a}\right)$ for all $a \in A$. Value distribution studies the magnitude of the inverse family $\xi_{f}=\left\{F_{a}\right\}_{a \in A}$. Do the First Main Theorem, the Mean Value Theorem, and the Defect Relation hold? Certainly some additional assumptions have to be made. We shall sketch a basic procedure underlying practically all derivations of a First Main Theorem.

Inspired by (1.1), we require the existence of a form $\Omega$ of bidegree $(s, s)$ and class $C^{\infty}$ on $N$ and the existence of a form $\Lambda_{a}$ of class $C^{\infty}$ and of bidegree $(s-1, s-1)$ on $N-E_{a}$ for each $a \in A$, such that $\Lambda_{a}$ has residue 1 on $E_{a}$ and such that

$$
d d^{c} \Lambda_{a}=\Omega
$$

on $N-E_{a}$. The following examples show that these assumptions can be satisfied in many cases.

EXAMPLE 1. Let $\&$ be the family of complex projective planes of dimension 
$n-s$ in the $n$-dimensional complex projective space $\mathbf{P}_{n}$. Let $\omega$ be the exterior form of the Fubini-Study Kaehler metric on $\mathbf{P}_{n}$. Take $\Omega=\omega^{s} \geqslant 0$. Then Chern [5] and Levine [17] construct $\Lambda_{a} \geqslant 0$ explicitly.

ExAMPLe 2. Let $\&$ be the point family, i.e., $N=A$ and $E_{a}=\{a\}$ for all $a \in N$. Assume $A$ is a compact Kaehler manifold. Let $\Omega$ be the Kaehler volume form of $A$ normalized such that $\int_{A} \Omega=1$. Hodge theory provides $\Lambda_{a} \geqslant 0$. See Wu [32], Hirschfelder [14], and Stoll [24].

Let $X$ and $Y$ be complex spaces. Let $h: X \rightarrow Y$ be a holomorphic map. Then $h$ is said to be projective if for every $z \in X$ open neighborhoods $U$ of $z$ and $V$ of $h(z)$ exist such that there is a complex space $W$ and a biholomorphic map $\alpha: U \rightarrow V \times W$ such that $h \mid U=P \circ \alpha$ where $P: V \times W \rightarrow V$ is the projection. Assume that $h$ is proper, projective and has pure fiber dimension $q$. Then the fiber integration operator $h_{*}$ is defined and associates to each form $\chi$ of bidegree $(k, l)$ and class $C^{\infty}$ on $X$ a form $h_{*} \chi$ of bidegree $(k-q, l-q)$ and of class $C^{\infty}$ on $Y$ provided $k \geqslant q$ and $l \geqslant q$. See Tung [29].

EXAMPLe 3. The family $E=\left\{E_{a}\right\}_{a \in A}$ is said to be admissible if the following conditions are satisfied.

(i) The index set $A$ is a connected, complex Kaehler manifold.

(ii) The incidence set $S=\left\{(x, a) \in M \times A \mid x \in E_{a}\right\}$ is analytic.

(iii) The projections $\tau: S \rightarrow M$ and $\pi: S \rightarrow a$ are surjective and $\tau$ is proper and projective.

Determine $\Omega(A)$ and $\Lambda_{a}(A)$ for the point family on $A$ as in Example 2. Then $\Omega=\tau_{*} \pi^{*} \Omega(A) \geqslant 0$ and $\Lambda_{a}=\tau_{*} \pi^{*}\left(\Lambda_{a}(A)\right) \geqslant 0$ satisfy assumption (2.1). Also $\tau: \pi^{-1}(a) \rightarrow E_{a}$ is biholomorphic. See Tung [29]. Also observe that the point family in Example 2 is admissible.

We return to the general situation and assume (2.1). Also suppose that $F_{a}$ is either empty or has pure dimension $m-s$ if $a \in A$. Then (2.1) pulls back to $d d^{c} f^{*}\left(\Lambda_{a}\right)=f^{*}(\Omega)$ on $M-F_{a}$. Let $\varphi$ be a form of class $C^{\infty}$ and of bidegree $(m-s, m-s)$ on $M$. Assume $\varphi$ has compact support. The singularity of $f^{*}\left(\Lambda_{a}\right)$ on $F_{a}$ is so weak that Stokes' Theorem applies:

$$
\int_{M} f^{*}\left(\Lambda_{a}\right) \wedge d d^{c} \varphi=-\int_{M} d f^{*}\left(\Lambda_{a}\right) \wedge d^{c} \varphi
$$

For degree reasons $d f^{*}\left(\Lambda_{a}\right) \wedge d^{c} \varphi=d \varphi \wedge d^{c} f^{*}\left(\Lambda_{a}\right)$. A second application of Stokes' Theorem leaves a residue

$$
\begin{aligned}
\int_{M} f^{*}\left(\Lambda_{a}\right) \wedge d d^{c} \varphi & =-\int_{M} d\left(\varphi \wedge d^{c} f^{*}\left(\Lambda_{a}\right)\right)+\int_{M} \varphi \wedge d d^{c} f^{*}\left(\Lambda_{a}\right) \\
& =-\int_{F_{a}} \theta_{f a} \varphi+\int_{M} \varphi \wedge f^{*}(\Omega) .
\end{aligned}
$$

Here $\theta_{f a}$ is an integral-valued nonnegative multiplicity function. The unintegrated First Main Theorem is obtained:

$$
\int_{M} f^{*}(\Omega) \wedge \varphi=\int_{F_{a}} \theta_{f a} \varphi+\int_{M} f^{*}\left(\Lambda_{a}\right) \wedge d d^{c} \varphi
$$

The integral on the left does not depend on $a$. So (2.2) can be viewed as a preservation principle. Also (2.2) can be considered as an identity between currents. Let us consider two applications of (2.2). 
1. Application. Assume $M=N$ is compact and $f$ is the identity. Then $\theta_{f a}=1$ on $E_{a}$. Suppose $d \varphi=0$. Then $\int_{N} \Omega \wedge \varphi=\int_{E_{a}} \varphi$. Therefore $\Omega$ is the Poincaré dual of $E_{a}$ for each $a \in A$.

2. Application. Take $N=\mathbf{C}$ and $s=1$. Then $f$ is a holomorphic function. Choose $\Lambda_{a}(z)=-\log |z-a|^{2}$. Then $d d^{c} \Lambda_{a} \equiv 0$. Hence $\Omega \equiv 0$. Therefore

$$
\int_{F_{a}} \theta_{f}^{a} \varphi=\int_{M} \log |f-a|^{2} \wedge d d^{c} \varphi
$$

Hence the current of integration over the zeroes of $f$ is the current $d d^{c}\left[\log |f|^{2}\right]$, a theorem due to Lelong [16].

Now assume $\Omega \geqslant 0$ and $\Lambda_{a} \geqslant 0$, which can be satisfied in many cases, for instance in the examples. Then (2.2) shall be used to estimate the volume of $F_{a}$. Still the requirements that $\varphi$ ought to be the volume element on each $F_{a}$ and that $\varphi$ has compact support are incompatible. We shall refine the method. Assume a form $\chi \geqslant 0$ of bidegree $(m-s, m-s)$ and class $C^{\infty}$ and with $d \chi=0$ is given on $M$. If $M$ is Kaehlerian, the $(m-s)$ th power of the Kaehler form will do. Let $G$ and $g$ be relatively compact, open subsets of $M$ with smooth boundaries $\Gamma=\partial G$ and $\gamma=\partial g$. Assume $\bar{g} \subset G$. Let $\psi: M \rightarrow \mathbf{R}$ be a continuous function such that $\psi \mid(M-G)=0$ and $\psi \mid \bar{g}=R \geqslant 0$ are constant. Assume $0 \leqslant \psi \leqslant R$ on $M$. Also assume that $\psi \mid(\bar{G}-g)$ is of class $C^{\infty}$. Then $(G, \psi)$ is called a condensor. The characteristic $T_{f}(G)$, the valence function $N_{f}(G, a)$, the outer compensation function $m_{f}(\Gamma, a)$, the inner compensation function $m_{f}(\gamma, a)$ are defined by

$$
\begin{aligned}
T_{f}(G) & =\int_{G} \psi f^{*}(\Omega) \wedge \chi \geqslant 0, \\
N_{f}(G, a) & =\int_{F_{a}} \theta_{f a} \psi \chi \geqslant 0, \\
m_{f}(\Gamma, a) & =\int_{\Gamma} f^{*}\left(\Lambda_{a}\right)\left(-d^{c} \psi\right) \wedge \chi \geqslant 0, \\
m_{f}(\gamma, a) & =\int_{\gamma} f^{*}\left(\Lambda_{a}\right)\left(-d^{c} \psi\right) \wedge \chi \geqslant 0,
\end{aligned}
$$

where the integrands are nonnegative. The deficit is defined by

$$
D_{f}(G, a)=\int_{G} f^{*}\left(\Lambda_{a}\right) \wedge\left(-d d^{c} \psi\right) \wedge \chi
$$

In general the sign of the integrand of $D_{f}(G, a)$ is not fixed. If the proof of (2.2) is repeated with due regard to boundary integrals, the First Main Theorem is obtained:

$$
T_{f}(G)=N_{f}(G, a)+m_{f}(\Gamma, a)-m_{f}(\gamma, a)-D_{f}(G, a) .
$$

Assume that the index set $A$ is a compact Kaehler manifold with volume elements $\Omega(A)$ such that $\int_{A} \Omega(A)=1$. Then $I(h)=\int_{A} h \Omega(A)$ is the average of $h$ on $A$. Define $m_{f}(\Gamma)=I\left(m_{f}(\Gamma, a)\right)$ and $m_{f}(\gamma)=I\left(m_{f}(\gamma, a)\right)$. Also define $D_{f}(G)=I\left(D_{f}(G, a)\right)$. Then the Mean Value Theorem

$$
T_{f}(G)=I\left(N_{f}(G, a)\right)
$$

is equivalent to 


$$
D_{f}(G)=m_{f}(\Gamma)-m_{f}(\gamma)
$$

by (2.3). The Mean Value Theorem may not hold. In Example 1, the identity (2.5) can be proven directly by an exchange of integrals. See [22] and [23]. In Example 3, we proceed as follows: Let $(Q, \tilde{\tau}, \tilde{f})$ be the relative product of $f$ and $\tau$. Define $\hat{f}=\pi \circ \tilde{f}$. Then $\hat{f}$ maps $\hat{f}^{-1}(a)$ biholomorphically onto $F_{a}$. Also $\theta_{f a}(x)=1$ for all $x \in F_{a}$ and almost all $a \in A$. Moreover,

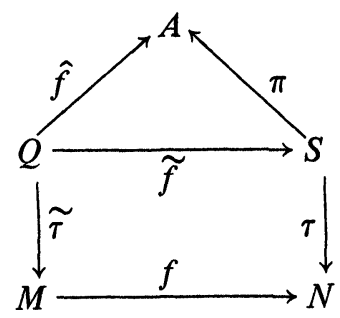

$$
f^{*}(\Omega)=f^{*} \tau_{*} \pi^{*}(\Omega(A))=\tilde{\tau}_{*} \tilde{f}^{*} \pi^{*}(\Omega(A))=\tilde{\tau}_{*} \hat{f}^{*}(\Omega(A))
$$

Hence,

$$
\begin{aligned}
T_{f}(G) & =\int_{M} f^{*}(\Omega) \wedge \psi \chi=\int_{M} \tilde{\tau}_{*} \hat{f}^{*}(\Omega(A)) \wedge \psi \chi \\
& =\int_{Q} \hat{f}^{*}(\Omega(A)) \wedge \tilde{\tau}^{*}(\psi \chi)=\int_{A} \hat{f}_{*} \tilde{\tau}^{*}(\psi \chi) \Omega(A) \\
& =\int_{A}\left(\int_{F_{a}} \psi \chi\right) \Omega(A)=I\left(N_{f}(G, a)\right) .
\end{aligned}
$$

So the Mean Value Theorem holds for admissible families.

Assume (2.4). Define $B=\left\{a \in A \mid F_{a} \neq \varnothing\right\}$. Then $0 \leqslant b_{f}=\int_{B} \Omega(A) \leqslant 1$ is the probability that $f(M)$ intersects $E_{a}$. Define

$$
D_{f}(G, a)^{+}=\int_{G} \operatorname{Max}\left(0, f^{*}\left(\Lambda_{a}\right) \wedge d d^{c} \psi \wedge \chi\right)
$$

and $D_{f}(G)^{+}=I\left(D_{f}(G, a)^{+}\right)$. If $a \in A-B$, then $N_{f}(G, a)=0$. Hence,

$$
N_{f}(G, a) \leqslant T_{f}(G)+m_{f}(\gamma, a)+D_{f}(G, a)^{+}
$$

implies

$$
T_{f}(G)=\int_{B_{f}} N_{f}(G, a) \Omega(A) \leqslant b_{f} T_{f}(G)+m_{f}(\gamma)+D_{f}(G)^{+} .
$$

If $T_{f}(G)>0$, then

$$
\left(1-b_{f}\right) \leqslant\left(m_{f}(\gamma)+D_{f}(G)^{+}\right) / T_{f}(G) .
$$

Keep $g$ fixed. Let $G$ exhaust $M$ with convenient $\psi=\psi_{G}$. If $T_{f}(G)$ grows more quickly than $m_{f}(\gamma)+D_{f}(G)^{+}$, we obtain $b_{f}=1$. Then $f(M) \cap E_{a} \neq \varnothing$ for almost all $a \in A$ which is called a Casorati-Weierstrass Theorem.

At this point, more accurate choices have to be made which lead to specific situations. Especially, $(G, g, \psi)$ has to be chosen. Some of these choices shall be mentioned here. 
1. Choice. Take $s=1$. The closed $C^{\infty}$-form $\chi$ has bidegree $(m-1, m-$ 1). Assume $\chi>0$. Then choose $\psi=\psi_{G}$ and $R=R(G)>0$ such that $d d^{c} \psi$ $\wedge \chi=0$ on $G-\bar{g}$, such that $\psi \mid \Gamma=0$ and $\psi \mid \gamma=R(G)$ with $\int_{\Gamma}\left(-d^{c} \psi_{G}\right) \wedge \chi$ $=1$. This Dirichlet problem has one and only one solution. Then $D_{f}(G, a)=$ $0=D_{f}(G, a)^{+}$and $D_{f}(G)=0=D_{f}(G)^{+}$. The First Main Theorem is freed from the deficit. A Casorati-Weierstrass Theorem and a Defect Relation can be obtained for the hyperplane family. See Weyl [30] if $m=1$ and [21] if $m>1$.

In the other cases, a nonnegative function $\tau: M \rightarrow \mathbf{R}$ of class $C^{\infty}$ is used. If $K \subseteq M$ and $r \geqslant 0$, define

$$
\begin{aligned}
& K[r]=\left\{z \in K \mid \tau(z) \leqslant r^{2}\right\}, \\
& K(r)=\left\{z \in K \mid \tau(z)<r^{2}\right\}, \\
& K\langle r\rangle=\left\{z \in K \mid \tau(z)=r^{2}\right\} .
\end{aligned}
$$

Then $\tau$ is said to be an exhaustion if $M[r]$ is compact for all $r \geqslant 0$. We call $M[r]$ the closed pseudoball, $M(r)$ the open pseudoball, and $M\langle r\rangle$ the pseudosphere. Let $u$ be an increasing function. Take $0 \leq r_{0}<r$ and define $G=$ $M(r)$ and $g=M\left(r_{0}\right)$ and $\psi_{r}=u\left(r^{2}\right)-u \circ \tau$ on $\bar{G}-g$. Now, other choices can be made.

2. CHOICE. Take an exhaustion $\tau$ with $d d^{c} \tau \leqslant 0$ outside $M\left[r_{0}\right] \neq \varnothing$. Then $\tau$ is called pseudoconcave. Take $u(x)$. $\equiv x$. Then $D_{f}(G, a) \leqslant 0$. Hence $D_{f}(G) \leqslant$ $D_{f}(G)^{+}=0$. Also $m_{f}(\gamma)$ is constant. If $T_{f}(G) \rightarrow \infty$ for $r \rightarrow \infty$, a CasoratiWeierstrass Theorem holds. See Chern [5], Bott and Chern [2], and Cowen [6].

3. CHOICE. Take an exhaustion $\tau$ with $d d^{c} \tau \geqslant 0$. Then $\tau$ and $M$ are called pseudoconvex. Take $u(x) \equiv x$. Then $D_{f}(G, a)=D_{f}(G, a)^{+} \geqslant 0$ and $D_{f}(G)=$ $D_{f}(G)^{+} \geqslant 0$. A Casorati-Weierstrass Theorem follows if $D_{f}(M(r)) / T_{f}(M(r))$ $\rightarrow 0$ for $r \rightarrow \infty$. In many cases $D_{f}(M(r))$ can be computed. Observe that $M$ is a Stein manifold if and only if a pseudoconvex exhaustion $\tau$ exists with $d d^{c} \tau>0$ on $M$. Stein manifolds are the most important noncompact complex manifolds and value distribution applies to them.

4. CHоICE. Take an exhaustion $\tau$ such that $d d^{c} \log \tau \geqslant 0$ and $\left(d d^{c} \log \tau\right)^{m} \equiv$ 0 . Also $\left(d d^{c} \tau\right)^{m} \not \equiv 0$ and $M[0]$ of measure zero are required. Then $\tau$ is called a parabolic exhaustion and $M$ is called parabolic. Take $u=-\left[(2 m-2) x^{m}\right]^{-1}$. A Casorati-Weierstrass Theorem and a defect relation hold. For more details see below.

3. Some historical remarks. In 1938, H. Kneser [15] established the First Main Theorem for meromorphic functions on $\mathbf{C}^{m}$. About this time, Ahlfors [1], H. Weyl and J. Weyl [30] proved the First Main Theorem and the Defect Relation for holomorphic maps from Riemann surfaces into the complex projective space $\mathbf{P}_{n}$ and for the hyperplane family. In 1970, Wu [33] renovated this theory. In 1953-1954, these theories were united and expanded to meromorphic maps of complex manifolds into $\mathbf{P}_{n}$ for the hyperplane family; see [21]. The First Main Theorem and the Defect Relation were proved in [21] under Choice 1. In 1960, Levine [17] and Chern [5] established the First Main Theorem for holomorphic maps of $\mathbf{C}^{m}$ into $\mathbf{P}_{n}$ for the family of $p$-planes in $\mathbf{P}_{n}$. Here the deficit term first appears. In 1967-1969, this 
theory was extended to holomorphic maps of pseudoconvex manifolds into $\mathbf{P}_{n}$; see [22] and [23]. Up to date, no Defect Relation for the $p$-plane family has been found and this remains one of the most difficult open problems in this subject matter.

Around 1968, the First Main Theorem for admissible families was proved. $\mathrm{Wu}$ [32] treated the point family only. Hirschfelder [13] required that the parameter space $A$ is homogeneous. In [24], the general case was established. In 1973 Tung [29] extended this theory to complex spaces and meromorphic maps. In 1973, Ahlfors estimates were established in [26] for meromorphic maps of pseudoconvex spaces into $\mathbf{P}_{n}$ for the hyperplane family. The results were applied to the Bezout Problem. In 1974, Murray [19] completed these investigations by establishing a Defect Relation.

In 1965, Bott and Chern [2] created a new theory of value distribution for holomorphic sections in a holomorphic vector bundle. Let $M$ and $N$ be complex spaces of pure dimensions $m$ and $n$ respectively. Let $f: M \rightarrow N$ be a holomorphic map. Let $E$ be a holomorphic vector bundle of fiber dimension $p$ over $N$. For each holomorphic section $s$ of $E$, let $Z(s)$ be the zero set of $s$. Assume, a finite-dimensional vector space $V$ of holomorphic sections of $E$ over $N$ is given, which generates each fiber of $E$ over $\mathbf{C}$. Then $E=$ $\{Z(s)\}_{s \in V-\{0\}}$ is the family considered on $N$. Bott and Chern proved the First Main Theorem and the Casorati-Weierstrass Theorem on pseudoconcave manifolds if $M=N$ and $p=n$ and if $f$ is the identity. In 1970, an indication was given in [25] how to extend the Bott-Chern theory to Stein manifolds if $1 \leqslant p \leqslant n$. In 1971, Cowen [6] introduced Schubert zeroes of vector bundles and proved the First Main Theorem and a CasoratiWeierstrass Theorem on pseudoconcave manifolds for Schubert zeroes. In all this $M=N$ and $f$ is the identity. The general case $f: M \rightarrow N$ is investigated in [28] on pseudoconvex manifolds.

During the last six years, Phillip Griffiths and his school considered the case of a line bundle $(p=1)$ and introduced a wealth of new ideas into value distribution. One of the main accomplishments is a new proof of the Defect Relation. The previous proofs by Ahlfors, Weyl, Stoll, and later ones by Murray and Wong use so-called associated maps. In 1972, Carlson and Griffiths [4] constructed a singular volume form $\xi$ on $N$ such that Ric $\xi \geqslant 0$ and (Ric $\xi)^{n} \geqslant \xi$. This form yields the Defect Relation. In 1973, Griffiths and King [12] extended this theory to holomorphic maps $f: M \rightarrow N$, where $M$ is affine algebraic and $N$ is projective algebraic. As above, a holomorphic line bundle $L$ over $N$ is given and $\&$ is the family of zeroes of holomorphic sections of $L$. An important assumption is $\operatorname{Rank} f=\operatorname{dim} N \leqslant \operatorname{dim} M$. In 1974-1975 this theory of Carlson, Griffiths and King was made intrinsic and extended to parabolic manifolds $M$; see [27]. An outline of this extended theory follows. For meromorphic maps from parabolic manifolds into projective space Wong [31] proves a Defect Relation without dimension restrictions using associated maps.

4. Value distribution on parabolic spaces. Here, we will consider the Carlson-Griffiths-King theory [4], [12] as extended in [27]. This section is restricted to codimension 1 . Higher codimensions are treated in the next section. Let $M$ and $N$ be connected, complex manifolds of dimensions $m$ and 
$n$ respectively. Assume $N$ is compact, but $M$ is not compact. On $N$, a holomorphic line bundle $L$ is given. Let $f: M \rightarrow N$ be a holomorphic map. This situation will prevail through the remainder of this report.

Let $\kappa$ be a hermitian metric along the fibers of $L$. Let $c(L, \kappa)$ be the associated Chern form. If $U \neq \varnothing$ is open in $M$ and if $s: U \rightarrow L$ is a holomorphic section without zeroes, then

$$
c(L, \kappa)=-d d^{c} \log |s|_{\kappa}^{2}
$$

on $U$. Assume $L$ is nonnegative, that is, $c(L, \kappa) \geqslant 0$ for a choice of $\kappa$ and choose $\kappa$ so.

Since $N$ is compact, the vector space $V$ of all holomorphic sections of $L$ over $N$ has finite dimension $k+1$. Assume $k \geqslant 0$. If $0 \neq \mathfrak{a} \in V$, define $\mathbf{P}(\mathfrak{a})=\mathbf{C a}$. If $A \subseteq V$, define $\mathbf{P}(A)=\{\mathbf{P}(\mathfrak{a}) \mid 0 \neq \mathfrak{a} \in A\}$. Then $\mathbf{P}(V)$ is the complex projective space associated to $V$ and $\mathbf{P}: V-\{0\} \rightarrow \mathbf{P}(V)$ is holomorphic. If $a \in \mathbf{P}(V)$, then $a=\mathbf{P}(\mathfrak{a})$ for some $0 \neq \mathfrak{a} \in V$ and $E_{L}[a]=Z(\mathfrak{a})$ does not depend on the choice of $a$ but on $a$ only. The set $E_{L}[a]$ is empty or pure $(n-1)$-dimensional. Also $a$ is called a projective section and $E_{L}[a]$ its zero set. Then $E=\left\{E_{L}[a]\right\}_{a \in \mathbf{P}(V)}$ is the value distribution family to be considered on $N$.

Take a hermitian metric $l$ on $V$. If $x \in N$ and $a \in \mathbf{P}(V)$, choose $a \in$ $\mathbf{P}^{-1}(a)$ with $|a|_{l}=1$. Then $0 \leqslant\|a, x\|_{\kappa}=|\mathfrak{a}(x)|_{\kappa}$ does not depend on the choice of $a$. Then $\kappa$ is said to be distinguished if $\|a, x\|_{\kappa} \leqslant 1$ for all $a \in \mathbf{P}(V)$ and $x \in N$. If $\kappa$ is not already distinguished a constant $\lambda>0$ exists such that $\lambda \kappa$ is distinguished. Observe $c(L, \lambda \kappa)=c(L, \kappa) \geqslant 0$. Hence, w.l.o.g. $\kappa$ can be assumed to be distinguished. We have

$$
c(L, \kappa)=-d d^{c} \log \|a, x\|_{\kappa}^{2}
$$

on $N-E_{L}[a]$. The base point set

$$
E_{L}[\infty]=\bigcap_{a \in \mathbf{P}(V)} E_{L}[a]
$$

is analytic and $N_{\infty}=N-E_{L}[\infty]$ is open and dense. For $x \in N$, the evaluation map $\eta_{x}: V \rightarrow L_{x}$ is defined by $\eta_{x}(\mathfrak{a})=\mathfrak{a}(x)$ for all $\mathfrak{a} \in V$. Let $S_{x}$ be the kernel of $\eta_{x}$ and let $\left(S_{x}\right)^{\perp}$ be the orthogonal complement of $S_{x}$ by $l$. If and only if $x \in N_{\infty}$, the map $\eta_{x}$ is surjective and $\eta_{x}:\left(S_{x}\right)^{\perp} \rightarrow L_{x}$ is an isomorphism. In this case the restriction of $l$ to $\left(S_{x}\right)^{\perp}$ carries over to a hermitian metric $l_{x}$ on $L_{x}$. This defines a hermitian metric $l$ along the fibers of $L \mid N_{\infty}$. Then $c(L, l) \geqslant 0$ on $N_{\infty}$. Also $0 \leqslant\|a, x\|_{l} \leqslant 1$ if $a \in \mathbf{P}(V)$ and $x \in N_{\infty}$.

Also $M$ needs a measure. Let $\tau \geqslant 0$ be a nonnegative function of class $C^{\infty}$ on $M$. If $K \subseteq M$, define $K[r], K(r)$ and $K\langle r\rangle$ as in (2.8), (2.9) and (2.10). Then $\tau$ is said to be a parabolic exhaustion of $M$ if the following conditions are satisfied:

(1) For each $r \geqslant 0$, the closed pseudoball $M[r]$ is compact.

(2) The center $M[0]$ has measure zero.

(3) On $M-M[0]$, assume $\omega=d d^{c} \log \tau \geqslant 0$. Then $v=d d^{c} \tau \geqslant 0$ on $M$.

(4) Assume $v^{m} \not 0$ but $\omega^{m} \equiv 0$.

Then $M(r) \neq \varnothing$ if $r>0$. Also $M[0] \neq \varnothing$. Let $\Re_{\tau}$ be the set of all $r>0$ such that $(d \tau)(x) \neq 0$ for all $x \in M\langle r\rangle$. If $r \in \Re_{\tau}$, then $M\langle r\rangle$ is the oriented boundary manifold of $M(r)$. For $q \geqslant 0$ define 


$$
\sigma_{q}=d^{c} \log \tau \wedge \omega^{q} \text { and } \sigma=\sigma_{m-1} .
$$

Let $j_{r}: M\langle r\rangle \rightarrow M-M[0]$ be the inclusion. If $r \in \Re_{\tau}$, then $j_{r}^{*}(\sigma) \geqslant 0$ and the pseudosphere $M\langle r\rangle$ has constant volume

$$
\varsigma=\int_{M\langle r\rangle} \sigma>0 .
$$

For all $r>0$, the volume of the pseudoball is given by

$$
\int_{M[r]} v^{m}=\int_{M(r)} v^{m}=s r^{2 m}
$$

Now, $M$ together with $\tau$ is called a parabolic manifold, also denoted by $(M, \tau)$. They are many examples of parabolic manifolds. For instance:

1. EXAMPLe. $\left(\mathbf{C}^{m}, \tau\right)$ is parabolic with $\tau(z)=|z|^{2}$.

2. EXAMPLE. Let $M$ and $\tilde{M}$ be connected, noncompact, complex manifolds of dimension $m$. Let $\beta: \tilde{M} \rightarrow M$ be a proper, surjective, holomorphic map. Let $\tau$ be a parabolic exhaustion of $M$. Then $\tau \circ \beta$ is a parabolic exhaustion of $\tilde{M}$.

3. EXAMPLE. Any affine algebraic manifold is parabolic.

4. EXAMPLE. Let $\left(M_{1}, \tau_{1}\right)$ and $\left(M_{2}, \tau_{2}\right)$ be parabolic manifolds. Define $M=M_{1} \times M_{2}$. Let $\pi_{j}: M \rightarrow M_{j}$ be the projections for $j=1,2$. Then $\tau=$ $\tau_{1} \circ \pi_{1}+\tau_{2} \circ \pi_{2}$ is a parabolic exhaustion of $M$. Hence the product of parabolic manifolds is parabolic.

5.EXAMPLE. Let $B$ be a compact, connected complex manifold of dimension $m-1$. Let $M$ be a holomorphic line bundle over $B$ with a hermitian metric $\tau$ along the fibers of $M$. Assume $c(M, \tau) \leqslant 0$ on $M$ and $c(M, \tau)\left(x_{0}\right)<0$ at some point $x_{0} \in M$. Then $(M, \tau)$ is parabolic.

6. EXAMPLE. A noncompact Riemann surface is parabolic if and only if it belongs to the class $\mathfrak{D}_{g}$, that is, any subharmonic function bounded above is constant.

7. EXAMPLE. $(\mathbf{C}-\mathbf{Z}) \times \mathbf{C}^{m}$ is parabolic but not affine algebraic.

A nonnegative divisor $\nu$ can be defined in various ways. Since $M$ is a manifold, $\nu$ can be identified with its multiplicity function. A nonnegative function $\nu: M \rightarrow \mathbf{Z}$ is said to be a nonnegative divisor, if $\nu$ is locally the zero multiplicity of a local holomorphic function. Then $A=\operatorname{supp} \nu$ is empty or a pure $(m-1)$-dimensional analytic set. Also $\nu$ is locally constant on the set of simple points of $A$. For $r>0$, the counting function of $\nu$ is defined by

$$
n_{\nu}(r)=r^{2-2 m} \int_{A[r]} \nu v^{m-1} \geqslant 0 .
$$

The function increases. Hence $n_{\nu}(r) \rightarrow n_{\nu}(0) \geqslant 0$ for $r \rightarrow 0$ exists. Then

$$
n_{\nu}(r)=\int_{A[r]} \nu \omega^{m-1}+n_{\nu}(0) \text {. }
$$

For $0<s<r$, the valence function of $\nu$ is defined by

$$
N_{\nu}(r, s)=\int_{s}^{r} n_{\nu}(t) \frac{d t}{t} .
$$

Let $f: M \rightarrow N$ be a holomorphic map. For simplicity, assume that $f(M)$ is not contained in $E_{L}[a]$ if $a \in \mathbf{P}(V)$. The spherical image is defined by 


$$
A_{f}(r, L, \kappa)=r^{2-2 m} \int_{M[r]} f^{*}(c(L, \kappa)) \wedge v^{m-1} \geqslant 0 .
$$

The function increases. The limits $A_{f}(r, L, \kappa) \rightarrow A_{f}(0, L, \kappa)$ for $r \rightarrow 0$ and $A_{f}(r, L, \kappa) \rightarrow A_{f}(\infty, L, \kappa) \leqslant \infty$ for $r \rightarrow \infty$ exist. Then

$$
A_{f}(r, L, \kappa)=\int_{M[r]} f^{*}(c(L, \kappa)) \wedge \omega^{m-1}+A_{f}(0, L, \kappa) .
$$

For $0<s<r$, the characteristic of $f$ is defined by

$$
T_{f}(r, s, L, \kappa)=\int_{s}^{r} A_{f}(t, L, \kappa) \frac{d t}{t} \geqslant 0 .
$$

If $f$ is not constant and $c(L, \kappa)>0$ on $N$, then

$$
\begin{gathered}
T_{f}(r, s, L, \kappa) \rightarrow \infty \text { for } r \rightarrow \infty, \\
T_{f}(r, s, L, \kappa) /(\log r) \rightarrow A_{f}(\infty, L, \kappa)>0 \text { for } r \rightarrow \infty .
\end{gathered}
$$

In any case, assume $A_{f}(\infty, L, \kappa)>0$ which implies (4.5) and (4.6). For $a \in \mathbf{P}(V)$ and $r \in \Re_{\tau}$, the compensation function is defined by

$$
m_{f}(r, a, L, \kappa)=\int_{M\langle r\rangle} \log \frac{1}{\|a, f\|_{\kappa}} \sigma \geqslant 0 .
$$

The function extends to a continuous function of $r$ for all $r>0$. For $a \in \mathbf{P}(V)$, the map $f$ defines a nonnegative divisor $\theta_{f a}$ with support $F_{a}=$ $f^{-1}\left(E_{L}[a]\right)$. The counting function of $\theta_{f a}$ is denoted by $n_{f}(r ; a, L)$ and the valence function by $N_{f}(r, s ; a, L)$. For $0<s<r$ the First Main Theorem holds:

$$
T_{f}(r, s, L, \kappa)=N_{f}(r, s ; a, L)+m_{f}(r ; a, L, \kappa)-m_{f}(s ; a, L, \kappa) .
$$

Assume $F_{\infty}=f^{-1}\left(E_{L}[\infty]\right)$ has at most dimension $m-2$. Let $\varphi$ be the exterior form of the Fubini-Study-Kaehler metric defined by $l$ on $\mathbf{P}(V)$. Then $\mathbf{P}(V)$ has volume 1 . Unfortunately, the Mean Value Theorem does not hold. Fortunately, the statements above remain correct if $\kappa$ is replaced by $l$, although $l$ is defined over $N_{\infty}$ only. Then the Mean Value Theorem holds:

$$
T_{f}(r, s, L, l)=\int_{\mathbf{P}(V)} N_{f}(r, s ; a, L) \varphi(a)^{k} .
$$

Assume (4.5) for $\kappa$ and $l$. Define the Nevanlinna defect of $a \in \mathbf{P}(V)$ by

$$
0 \leqslant \delta_{f}(a, L)=\liminf _{r \rightarrow \infty} \frac{m_{f}(r ; a, L, \kappa)}{T_{f}(r, s, L, \kappa)} .
$$

The First Main Theorem and (4.5) imply

$$
1 \geqslant \delta_{f}(a, L)=1-\limsup _{r \rightarrow \infty} \frac{N_{f}(r, s ; a, L)}{T_{f}(r, s, L, \kappa)} .
$$

Then $\delta_{f}(a, L)$ does not depend on $s$ and $\kappa$. However, if in (4.8) $\kappa$ is replaced by $l$, another defect $\delta_{f}^{0}(a, L)$ may be obtained. Of course if $E_{L}[\infty]=\varnothing$, then $\delta_{f}^{0}(a, L)=\delta_{f}(a, L)$. Also (4.9) holds for $l$. Hence $0 \leqslant \delta_{f}^{0}(a, L) \leqslant 1$. If $F_{a}=$ $\varnothing$, then $\delta_{f}^{0}(a, L)=\delta_{f}(a, L)=1$ since $N_{f}(r, s ; a, L)=0$. The Mean Value Theorem and Fatou's Lemma imply $I\left(\delta_{f}^{0}(a, L)\right)=0$. Hence $\delta_{f}^{0}(a, L)=0$ for 
almost all $a \in \mathbf{P}(V)$. Therefore $F(M) \cap E_{L}[a] \neq \varnothing$ for almost all $a \in \mathbf{P}(V)$. A Casorati-Weierstrass Theorem is obtained.

The results stated so far in this section extend to complex spaces and meromorphic maps. For the remainder of this section $M$ and $N$ have to be complex manifolds and $f$ is holomorphic. Now, the Second Main Theorem and the Defect Relation shall be stated, which requires a number of preparations.

To each form $\Omega>0$ of degree $2 m$ and class $C^{\infty}$ on $M$, a Ricci form Ric $\Omega$ of bidegree $(1,1)$ and class $C^{\infty}$ is assigned. Let $\alpha_{1}, \ldots, \alpha_{m}$ be local holomorphic coordinates on an open subset $U$ of $M$. Then a positive function $\Omega_{\alpha}$ of class $C^{\infty}$ exists on $U$ such that

$$
\Omega \mid U=\Omega_{\alpha} i^{m} d \alpha_{1} \wedge d \bar{\alpha}_{1} \wedge \ldots \wedge d \alpha_{m} \wedge d \bar{\alpha}_{m} .
$$

Then Ric $\Omega \mid U=d d^{c} \log \Omega_{\alpha}$ on $U$. For $0<s<r$, the Ricci function of $\Omega$ is defined by

$$
\operatorname{Ric}(r, s, \Omega)=\int_{s}^{r} t^{1-2 m} \int_{M[t]} \operatorname{Ric} \Omega \wedge v^{m-1} d t .
$$

Actually, $\operatorname{Ric}(r, s, \Omega)$ can be interpreted as the characteristic function of the canonical bundle $K_{M}$ of $M$ for a certain hermitian metric along $K_{M}$. However, $\operatorname{Ric}(r, s, \Omega)$ may not have a fixed sign. Ideally, we would like to consider $\operatorname{Ric}\left(r, s, v^{m}\right)$ but $v^{m}>0$ may not be true on all of $M$. Hence an alternative is needed. A function $v \geqslant 0$ of class $C^{\infty}$ on $M$ is defined by $v^{m}=v \Omega$. For almost all $0<s<r$, the Ricci function of $\tau$ is defined by

$$
\operatorname{Ric}_{\tau}(r, s)=\frac{1}{2} \int_{M\langle r\rangle} \log v \sigma-\frac{1}{2} \int_{M\langle s\rangle} \log v \sigma+\operatorname{Ric}(r, s, \Omega)
$$

and does not depend on the choice of $\Omega$. If $v^{m}>0$ on $M$, then $v=1$ implies $\operatorname{Ric}_{\tau}(r, s)=\operatorname{Ric}\left(r, s, v^{m}\right)$. If $\beta: M \rightarrow \mathbf{C}^{m}$ is a proper, surjective holomorphic map and if $\tau=|\beta|^{2}$, let $\mu$ be the branching divisor of $\beta$. Then

$$
\operatorname{Ric}_{\tau}(r, s)=N_{\mu}(r, s) \text {. }
$$

If the $(m-1)$-dimensional component of $\beta(\operatorname{supp} \mu)$ is algebraic, then

$$
N_{\mu}(r, s)=O(\log r) \text { for } r \rightarrow \infty .
$$

If $M=\mathbf{C}^{m}$, then $\operatorname{Ric}_{\tau}(r, s) \equiv 0$. If (4.5) holds, the Ricci defect is defined by

$$
R_{f}=\limsup _{r \rightarrow \infty} \frac{\operatorname{Ric}_{\tau}(r, s)}{T_{f}(r, s, L, \kappa)} \leqslant \infty
$$

and is independent of the choice of $s$ and $\kappa$.

Let $K_{M}$ and $K_{N}$ be the canonical bundles of $M$ and $N$ respectively. Let $K_{N}^{*}$ be the dual of $K_{N}$. The holomorphic map $f: M \rightarrow N$ pulls back $K_{N}$ and $K_{N}^{*}$ to $K_{N f}$ and $K_{N f}^{*}$, respectively, such that $K_{N f}^{*}$ is dual to $K_{N f}$. Call $K_{f}=K_{N} \otimes K_{N f}^{*}$ the Jacobian bundle of $f$. A global holomorphic section $F \not 0$ is called a Jacobian section of $f$. Let $\nu_{F}$ be the divisor of $F$. If (4.5) holds, the ramification defect is defined by

$$
0 \leqslant \Theta_{F}=\liminf _{r \rightarrow \infty} \frac{N_{\nu_{F}}(r, s)}{T_{f}(r, s, L, \kappa)} \leqslant \infty .
$$


A $K_{M}$-valued inner product $(\cdot, \cdot)$ exists between $K_{f}=K_{M} \otimes K_{N f}^{*}$ and $K_{N, f}$ Hence $F$ can be interpreted as an operator on certain forms. Let $\Omega^{n}(U)$ be the set of all holomorphic forms of bidegree $(n, 0)$ on the open subset $U$ of $N$. Assume $U_{f}=f^{-1}(U) \neq \varnothing$. Then $F$ acts as a homomorphism $F: \Omega^{n}(U) \rightarrow$ $\Omega^{m}\left(U_{f}\right)$. Take $\psi \in \Omega^{n}(U)$. Then $\psi$ is a holomorphic section of $K_{N}$ over $U$ which pulls back to a holomorphic section $\psi_{f}$ of $K_{N, f}$ over $U_{f}$. In general, $\psi_{f}$ is not the pullback $f^{*}(\psi)$ of forms. The inner product defines a holomorphic section $F[\psi]=\left(F, \psi_{f}\right)$ of $K_{M}$ over $U_{f}$ which is a holomorphic form of bidegree $(m, 0)$ on $U_{f}$. This action extends to forms of degree $2 n$. Let $A^{2 n}(U)$ be the forms of degree $2 n$ and class $C^{\infty}$ on $U$. Then $F$ acts as a homomorphism $F: A^{2 n}(U) \rightarrow A^{2 m}\left(U_{f}\right)$ such that the following condition is satisfied: For each integer $p \geqslant 0$ define

$$
i_{p}=(-1)^{p(p-1) / 2} p !(i / 2 \pi)^{p} .
$$

If $\varphi \in \Omega^{n}(U)$ and $\chi \in \Omega^{n}(U)$, then the action of $F$ on $A^{2 n}(U)$ is determined by

$$
F\left[i_{n} \varphi \wedge \bar{\chi}\right]=i_{m} F[\varphi] \wedge \operatorname{conj}(F[\chi])
$$

where conj denotes conjugation. If $0<\psi \in A^{2 n}(U)$, and if $Z(F)$ is the zero set of $F$, then $F[\psi]>0$ on $U_{f}-Z(F)$ and

$$
\operatorname{Ric} F[\psi]=f^{*}(\operatorname{Ric} \psi) \text { on } U_{f}-Z(F)
$$

Define $M^{+}=\{x \in M \mid v(x)>0\}$ and $M^{+}(r)=M^{+} \cap M(r)$. Then $F$ is said to be dominated by $\tau$ if for each $r>0$ there exists a minimal constant $Y(r) \geqslant 1$ such that for all open sets $U$ of $N$ with $M^{+}(r) \cap U_{f} \neq \varnothing$ and for all forms $\psi \geqslant 0$ of bidegree $(1,1)$ and class $C^{\infty}$ on $U$ the inequality

$$
n\left(F\left[\psi^{n}\right] / v^{m}\right)^{1 / n} v^{m} \leqslant Y(r) f^{*}(\psi) \wedge v^{m-1}
$$

holds on $U_{f} \cap M^{+}(r)$. The function $Y$ increases and is called the dominator of $F$. If $Y$ exists, then $\operatorname{dim} M \geqslant \operatorname{dim} N=\operatorname{rank} f$. If this necessary condition is satisfied, $Y$ exists under reasonable assumptions, for instance, if $v>0$ on $M$. For example, if $m=n$, then a Jacobian section dominated by $Y \equiv m$ is defined by $F[\varphi]=f^{*}(\varphi)$. For instance, if there exists a proper, surjective holomorphic map $\beta: M \rightarrow \mathbf{C}^{m}$ such that $\tau=|\beta|^{2}$, then a Jacobian section $F$ dominated by $\tau$ exists such that $Y \equiv m$. (2. Example of parabolic manifolds).

Let $F$ be Jacobian section of $f$ dominated by $\tau$ with dominator $Y$. Then the dominator defect is defined by

$$
0 \leqslant Y_{F}=\limsup _{r \rightarrow \infty} \frac{\log Y(r)}{T_{f}(r, s, L, \kappa)} \geqslant 0
$$

and does not depend on $s$ and $\kappa$.

Now, the Second Main Theorem can be formulated. For each index $j=1, \ldots, q$, let $L_{j}$ be a holomorphic line bundle on $N$ with a hermitian metric $\kappa_{j}$ along the fibers of $L_{j}$. Let $V_{j}$ be the vector space of all global holomorphic sections of $L_{j}$ and let $l_{j}$ be a hermitian metric on $L_{j}$. Take $a_{j} \in \mathbf{P}\left(V_{j}\right)$. Then $a_{1}, \ldots, a_{q}$ are said to be in general position (or have strictly normal crossings) if the following conditions are satisfied for each $x \in N$ : Let $v_{j}$ be a holomorphic section of $L_{j}$ over an open neighborhood $U_{j}$ of $x$ with 
$Z\left(v_{j}\right)=\varnothing$. Take $0 \neq \mathfrak{a}_{j} \in V$ with $\mathbf{P}\left(\mathfrak{a}_{j}\right)=a_{j}$. Holomorphic functions $w_{j}$ on $U$ are defined by $a_{j}=w_{j} v_{j}$. A sequence of integers $1 \leqslant \mu(1)<\cdots<\mu(t) \leqslant q$ exists such that $w_{\mu(p)}(x)=0$ for all $p=1, \ldots, t$, but $w_{j}(x) \neq 0$ if $j \neq \mu_{p}$ for all $p=1, \ldots, t$. Then $\left(d w_{\mu(1)} \wedge \ldots \wedge d w_{\mu(t)}\right)(x) \neq 0$, i.e., $w_{\mu(1)}, \ldots, w_{\mu(t)}$ is a subset of a set of coordinate functions at $x$.

Let $a_{1}, \ldots, a_{q}$ be in general position. Define $L=L_{1} \otimes \ldots \otimes L_{q}$ and $\kappa=\kappa_{1} \otimes \ldots \otimes \kappa_{q}$. Assume $c(L, \kappa)>0$. Take a positive integer $w$. A smallest integer $p \geqslant 0$ exists such that $L^{p} \otimes K_{N}^{w}$ is nonnegative. Assume $p<w$. Take $\varepsilon>0$ and $s>0$. Define $c_{2}=(n+\varepsilon) \varsigma$ and $c_{3}=2 \varepsilon s n$. Then there exist a constant $c_{1}=c_{1}(\varepsilon)>0$ and a measurable subset $\Delta=\Delta(\varepsilon)$ of $\mathbf{R}^{+}=\{x \in \mathbf{R} \mid x$ $>0\}$ such that the Second Main Theorem holds:

$$
\begin{aligned}
N_{\nu_{F}}(r, s) & +\left(1-\frac{p}{w}\right) T_{f}(r, s, L, \kappa) \leqslant \sum_{j=1}^{q} N_{f}\left(r, s ; a_{j}, L_{j}\right) \\
& +c_{1} \log T_{f}(r, s, L, \kappa)+c_{2} \log Y(r)+c_{3} \log r
\end{aligned}
$$

for all $r>s$ with $r \in \mathbf{R}^{+}-\Delta$ where $\int_{\Delta} x^{\varepsilon} d x<\infty$.

For the Defect Relation, assume $L_{1}=\cdots=L_{q}$. It may be helpful to recount the assumptions here. Let $N$ be a compact, connected complex manifold of dimension $n>0$. Let $L$ be a positive holomorphic line bundle on $N$. (Hence $N$ is projective algebraic.) Let $V$ be the vector space of global holomorphic sections of $L$ on $N$. Let $l$ be a hermitian metric on $V$. Let $\kappa$ be a distinguished hermitian metric along the fibers of $L$ with $c(L, \kappa)>0$. Let $p=\inf u / w$ with $u \geqslant 0, w>0$ such that $L^{u} \otimes K_{N}^{w}$ is nonnegative. Let $a_{1}, \ldots, a_{q}$ be given in $\mathbf{P}(V)$ such that $q>p$ and such that $a_{1}, \ldots, a_{q}$ are in general position for $L$. Let $(M, \tau)$ be a parabolic manifold of dimension $m>0$. Let $f: M \rightarrow N$ be a holomorphic map with a Jacobian section $F$ dominated by $\tau$. Let $Y$ be the dominator. Then the Defect Relation holds:

$$
\Theta_{F}+\sum_{j=1}^{q} \delta_{f}\left(a_{j}, L\right) \leqslant p+R_{f}+\varsigma n Y_{F} .
$$

Of course, (4.16) is only meaningful if $R_{f}<\infty$ and $Y_{F}<\infty$. If $N$ is the projective space and if $L$ is the hyperplane section bundle, then $p=n+1$. If $m=n=\operatorname{Rank} f$, then $F$ can be taken such that $Y \equiv m$. Also, if $m \geqslant n=$ Rank $f$ and if a proper, surjective, holomorphic map $\beta: M \rightarrow \mathbf{C}^{m}$ exists such that $\tau=|\beta|^{2}$, then $F$ can be chosen such that $Y \equiv m$. In both cases, $Y_{F}=0$. Hence

$$
\sum_{j=1}^{q} \delta_{f}\left(a_{j}, L\right) \leqslant p+R_{f}
$$

Observe, that (4.17) does not depend on the choice of $F$. Consider the covering case. Let $\mu$ be the branching divisor of $\beta$. Assume that the $(m-1)$ dimensional component of $\beta(\operatorname{supp} \mu$ ) is algebraic. (If $M$ is affine algebraic, all this can be realized.) Assume $f$ has transcendental growth, i.e., $A_{f}(\infty, L, \kappa)=$ $\infty$. Then (4.6), (4.10), (4.11) and (4.12) imply $R_{f}=0$. Hence

$$
\sum_{j=1}^{q} \delta_{f}\left(a_{j}, L\right) \leqslant p
$$

If $f(M) \cap E_{L}\left[a_{j}\right]=\varnothing$, then $\delta_{f}\left(a_{j}, L\right)=1$. Since $q>p$, at least one index $j$ 
exists such that $f(M) \cap E_{L}\left[a_{j}\right] \neq \varnothing$. The Borel Picard Theorem is proved.

The proof of the Defect Relation and (4.16) itself depends on the choice of the Jacobian section $F$. Moreover $F$ has to satisfy requirement (4.14) which implies $m \geqslant n=\operatorname{rank} f$. If $N=\mathbf{P}_{n}$ is the complex projective space and if $L$ is the hyperplane section bundle, a Defect Relation can be obtained using a holomorphic form $B \neq 0$ of bidegree $(m-1,0)$. Again, additional assumptions have to be made on $B$. Differentiation in the direction of $B$ is introduced and defines associated maps. No relation between $m$ and $n$ is required. Again, a ramification defect, a Ricci defect and a dominator defect appear. In both cases, the task of the operator $F$, respectively $B$, is to link $K_{N}$ with $K_{M}$. For details to the method using the form $B$ and associated maps see [1], [19], [21], [26], [30], [31] and [33], where [31] gives a Defect Relation quite similar to (4.16). For references to the method using Jacobian sections see [4], [12] and [27], where $F$ appears openly in [27] only. Also, applications are given in [4], [12] and [27].

5. Higher codimensions. The case of the common zero set of several holomorphic sections of the line bundle $L$ shall be considered. Only the First Main Theorem and a Casorati-Weierstrass Theorem have been established. Again, let $N$ be a connected, compact, complex manifold of dimension $n>0$. Let $L$ be a holomorphic line bundle on $N$ with a hermitian metric $\kappa$ along the fibers of $L$ such that $c(L, \kappa) \geqslant 0$. Let $V$ be the vector space of all global holomorphic sections of $V$. Then $\operatorname{dim} V=k+1<\infty$. Assume $k \geqslant 0$. Take a hermitian metric $l$ on $V$. Take $0 \leqslant p \leqslant k$. Then $\tilde{G}_{p}(V)=\left\{a_{0} \wedge \ldots \wedge\right.$ $\left.a_{p} \mid a_{j} \in V\right\}$ is analytic in $\bigwedge_{p+1} V$ and $G_{p}(V)=\mathbf{P}\left(\tilde{G}_{p}(V)\right.$ is a smooth, connected compact submanifold of dimension $d(p, n)=(n-p)(p+1)$ in $\mathbf{P}\left(\bigwedge_{p+1} V\right)$ called the Grassmann manifold of order $p$.

Take $a \in G_{p}(V)$. Then $a=\mathbf{P}(a)$ with $a=a_{0} \wedge \ldots \wedge a_{p}$. For $0 \leqslant \mu \leqslant p$, define $a_{\mu}=\mathbf{P}\left(\mathfrak{a}_{\mu}\right) \in \mathbf{P}(V)$ and

$$
\mathfrak{a}^{\mu}=a_{0} \wedge \ldots \wedge a_{\mu-1} \wedge a_{\mu+1} \wedge \ldots \wedge a_{p} .
$$

A section $\overrightarrow{\mathfrak{a}}^{\mu}$ of the trivial bundle $W_{p}=N \times \wedge_{p} V$ is defined by $\overrightarrow{\mathfrak{a}}^{\mu}(x)=$ $\left(x, \mathfrak{a}^{\mu}\right)$. A holomorphic section $\eta_{\mathfrak{a}}$ of $L \otimes W_{p}$ over $N$ is defined by

$$
\eta_{\mathfrak{a}}(x)=\sum_{\mu=0}^{p}(-1)^{\mu} \mathfrak{a}_{\mu}(x) \otimes \overrightarrow{\mathfrak{a}}^{\mu}(x) .
$$

Here $\eta_{\mathfrak{a}}$ depends on a but not on $a_{0}, \ldots, a_{p}$. Let $Z\left(\eta_{\mathfrak{a}}\right)$ be the zero set of $\eta_{\mathfrak{a}}$. The hermitian metric $l$ on $V$ defines a hermitian metric $l$ on $\bigwedge_{p} V$ and a hermitian metric $l$ along the fibers of the trivial bundle $W_{p}=N \times \wedge_{p} V$. Hence a hermitian metric $\kappa \otimes l$ is defined along the fibers of $L \otimes W_{p}$. Take $x \in M$, then

$$
0 \leqslant\|a ; x\|_{\kappa}=\left((\mathfrak{a})_{l}\right)^{-1}\left|\eta_{\mathfrak{a}}(x)\right|_{\kappa \otimes l}
$$

is defined and depends on $a$ but not on the choice of $a \in \mathbf{P}^{-1}(a)$. Also $E_{L}[a]=Z\left(\eta_{\mathfrak{a}}\right)$ depends on $a$ only. Obviously,

$$
E_{L}[a]=\left\{x \in M \mid\|a, x\|_{\kappa}=0\right\}=\bigcap_{\mu=0}^{p} E_{L}\left[a_{\mu}\right] .
$$


The Grassmann manifold $G_{k}(V)$ consists of one and only one point denoted by $\infty$. Then $E_{L}[\infty]$ is the base point set of $L$ consistent with (4.2) with $N_{\infty}=N-E_{L}[\infty] \neq \varnothing$. The hermitian metric $l$ on $V$ defines a hermitian metric $l$ along the fibers of $L \mid N_{\infty}$ as explained in $\S 4$. If $x \in N_{\infty}$, then $0 \leqslant\|a, x\|_{l} \leqslant 1$ and

$$
\|a, x\|_{\kappa}=\|a, x\|_{l}\|\infty, x\|_{\kappa} .
$$

Here $\|\infty, x\|_{\kappa}$ is called the deviation of $\kappa$. By multiplying $\kappa$ with a positive constant, $0 \leqslant\|\infty, x\|_{\kappa} \leqslant 1$ can be assumed w.l.o.g. Hence $\kappa$ is distinguished and $\|a, x\|_{\kappa} \leqslant 1$ for all $a \in G_{p}(V)$ and $x \in N$ by continuity. The form

$$
0 \leqslant \Phi_{L}[a]=c(L, \kappa)+d d^{c} \log \|a, x\|_{\kappa}^{2}
$$

is defined on $N-E_{L}[a]$ and is independent of $\kappa$ with $\Phi_{L}[a]^{p+1}=0$. On $N-E_{L}[a]$, the Chern-Levine form

$$
0 \leqslant \Lambda_{L}[a]_{\kappa}=-\log \|a, x\|_{\kappa}^{2} \sum_{\mu=0}^{p} \Phi_{L}[a]^{\mu} \wedge c(L, \kappa)^{p-\mu}
$$

is defined with

$$
d d^{c} \Lambda_{L}[a]_{\kappa}=c(L, \kappa)^{p+1} .
$$

Let $(M, \tau)$ be a parabolic manifold of dimension $m$. Assume $0 \leqslant p \leqslant k$ and $q=m-p-1 \geqslant 0$. Let $f: M \rightarrow N$ be a holomorphic map. For simplicity, assume that $F_{a}=f^{-1}\left(E_{L}[a]\right)$ is empty or has pure dimension $q$ for all $a \in G_{p}(V)$. For $r>0$ the spherical image of order $p$ is defined by

$$
A_{f}^{p}(r, L, \kappa)=r^{-2 q} \int_{M[r]} f^{*}\left(c(L, \kappa)^{p+1}\right) \wedge v^{q} \geqslant 0 .
$$

The function increases. The limits for $r \rightarrow 0$ and $r \rightarrow \infty$ exist and are denoted by $A_{f}(0, L, \kappa)$ and $A_{f}(\infty, L, \kappa)$, respectively. Then

$$
A_{f}^{p}(r, L, \kappa)=\int_{M[r]} f^{*}\left(c(L, \kappa)^{p+1}\right) \wedge \omega^{q}+A_{f}^{p}(0, L, \kappa) .
$$

For $0<s<r$, the characteristic of order $p$ is defined by

$$
T_{f}^{p}(r, s, L, \kappa)=\int_{s}^{r} A_{f}^{p}(t, L, \kappa) \frac{d t}{t} \geqslant 0 .
$$

The function increases and

$$
T_{f}^{p}(r, s, L, \kappa) /(\log r) \rightarrow A_{f}^{p}(\infty, L, \kappa) \text { for } r \rightarrow \infty .
$$

For each $a \in G_{p}(V)$, a multiplicity function $\theta_{f a} \geqslant 0$ is defined on $M$ with $F_{a}=\operatorname{supp} \theta_{f a}$ such that $\theta_{f a}$ is locally constant on the set of simple points of $F_{a}$. For $r>0$, the counting function of $f$ for $a \in G_{p}(V)$ is defined by

$$
n_{f}(r ; a, L)=r^{-2 q} \int_{F_{a}[r]} \theta_{f a} v^{2 q} \geqslant 0 .
$$

The function increases. Hence, $n_{f}(r ; a, L) \rightarrow n_{f}(0 ; a, L)$ for $r \rightarrow 0$. If $r>0$, then

$$
n_{f}(r ; a, L)=\int_{F_{a}[r]} \theta_{f a} \omega^{2 q}+n_{f}(0 ; a, L) .
$$


For $0<s<r$, the valence function of $f$ for $a \in G_{p}(V)$ is defined by

$$
N_{f}(r, s ; a, L)=\int_{s}^{r} n_{f}(t ; a, L) \frac{d t}{t} .
$$

Also define $M(s, r]=M[r]-M[s]$. Then the deficit of $f$ for $a$ is defined by

$$
D_{f}(r, s ; a, L, \kappa)=\frac{1}{2} \int_{M(s, r]} f^{*}\left(\Lambda_{L}[a]_{\kappa}\right) \wedge \omega^{q+1} \geqslant 0 .
$$

The function increases with $r$ and is semicontinuous from the right. For $r \in \Re_{\tau}$, the compensation function of $f$ for $a \in G_{p}(V)$ is defined by

$$
m_{f}(r ; a, L, \kappa)=\frac{1}{2} \int_{M\langle r\rangle} f^{*}\left(\Lambda_{L}[a]_{\kappa}\right) \wedge \sigma_{q} \geqslant 0
$$

where $\sigma_{q}$ is defined by (4.3). The function continues to a function which is semicontinuous from the right for all $r>0$. For $0<s<r$ the First Main Theorem holds:

$$
\begin{aligned}
T_{f}^{p}(r, s, L, \kappa)= & N_{f}(r, s ; a, L)+m_{f}(r ; a, L, \kappa) \\
& -m_{f}(s ; a, L, \kappa)-D_{f}(r, s ; a, L, \kappa) .
\end{aligned}
$$

Assume $\left.\left.F_{\infty}=f^{-1}\left(E_{L}\right] \infty\right]\right)$ has at most dimension $q-1$. Then $K$ can be replaced by $l$ in the preceding statements, although $l$ is singular over $E_{L}[\infty]$. The hermitian metric $l$ on $\bigwedge_{p+1} V$ defines a Fubini-Study-Kaehler metric on $\mathbf{P}\left(\bigwedge_{p+1} V\right)$ which restricts to a Kaehler metric on $G_{p}(V)$ whose exterior form is denoted by $\varphi_{p}$. Let $D(p, n)$ be the degree of the Grassmann manifold $G_{p}(V)$ in $\mathrm{P}\left(\bigwedge_{p+1} V\right)$. If $h$ is an integrable function on $G_{p}(V)$, its average is defined by

Then $I_{p}(1)=1$. Define

$$
I_{p}(h)=\frac{1}{D(p, n)} \int_{G_{p}(V)} h \varphi_{p}^{d(p, n)} .
$$

$$
\gamma_{p}=\frac{1}{2} \sum_{\nu=0}^{p} \sum_{\mu=1}^{k-p} \frac{1}{\nu+\mu}
$$

Then the following Mean Value Theorems hold:

$$
\begin{aligned}
I_{p}\left(n_{f}(r ; a, L)\right) & =A_{f}^{p}(r, L, l), \\
I_{p}\left(N_{f}(r, s ; a, L)\right) & =T_{f}^{p}(r, s ; L, l), \\
I_{p}\left(m_{f}(r ; a, L, l)\right) & =\gamma_{p} A_{f}^{p-1}(r ; L, l), \\
I_{p}\left(D_{f}(r, s ; a, L, l)\right) & =\gamma_{p}\left(A_{f}^{p-1}(r, L, l)-A_{f}^{P-1}(s, L, l)\right) .
\end{aligned}
$$

Assume $F_{a} \neq \varnothing$ for at least one $a \in G_{p}(V)$. Then $T_{f}^{p}(r, s ; L, l) \rightarrow \infty$ for $r \rightarrow \infty$. Define $\chi(a)=1$ if $F_{a} \neq \varnothing$ and $\chi(a)=0$ if $F_{a}=\varnothing$. Then $\chi$ is integrable over $G_{p}(V)$ with $0 \leqslant b_{f}(p)=I_{f}(\chi) \leqslant 1$. Here $b_{f}(p)$ is the probability that $f(M) \cap E_{L}[a] \neq \varnothing$. The First Main Theorem and the Mean Value Theorems imply easily that

$$
0 \leqslant 1-b_{f}(p) \leqslant \gamma_{p} A_{f}^{p-1}(r ; L, l) / T_{f}^{p}(r, s ; L, l)
$$

for all $0<s<r$. Hence, if 


$$
A_{f}^{p-1}(r ; L, l) / T_{f}^{p}(r, s ; L, l) \rightarrow 0 \text { for } r \rightarrow \infty,
$$

then $b_{f}(p)=1$, which means $f(M) \cap E_{L}[a] \neq \varnothing$ for almost all $a \in G_{p}(V)$, which is a Casorati-Wierstrass Theorem.

The results of this section hold even if $M$ and $N$ are complex spaces and if $f$ is meromorphic. Also the full force of the parabolic exhaustion $\tau$ is not needed. The assumption $\omega^{m} \equiv 0$ can be dropped, in which case $\tau$ is called a logarithmic pseudoconvex exhaustion. The results of this section extend also to the case where the line bundle $L$ is replaced by a vector bundle $E$ and a family of Schubert zeroes is considered. A Casorati-Weierstrass Theorem can be obtained; see [28]. For references to $\$ 5$ see [4], [12] and [27].

\section{REFERENCES}

1. L. V. Ahlfors, The theory of meromorphic curves, Acta. Soc. Sci. Fennicae, Nova Ser. A 3 (1941), no. 4, 1-31. 1941 MR 2, 357.

2. R. Bott and S. S. Chern, Hermitian vector bundles and the equidistribution of the zeroes of their holomorphic sections, Acta Math. 114 (1965), 71-112. MR 32 \#3070.

3. - Some formulas related to complex transgression, Essays on Topology and Related Topics. (Memoires dediés à Georges de Rham) Springer-Verlag, New York, 1970, pp. 44-57. MR 41 \# 9306.

4. J. Carlson and $\mathrm{Ph}$. Griffiths, $A$ defect relation for equidimensional holomorphic mappings between algebraic varieties, Ann. of Math. (2) 95 (1972), 557-584. MR 47 \#497.

5. S. S. Chern, The integrated form of the first main theorem for complex analytic mappings in several variables, Ann. of Math. (2) 71 (1960), 536-551. MR 23, \#A3276.

6. M. Cowen, Hermitian vector bundles and value distribution for Schubert cycles, Trans. Amer. Math. Soc. 180 (1973), 189-228. MR 48 \#11577.

7. M. Green, Holomorphic maps into complex projective space omitting hyperplanes, Trans. Amer. Math. Soc. 169 (1972), 89-103. MR 46 \# 7547.

8. Ph. Griffiths, Holomorphic mapping into canonical algebraic varieties, Ann. of Math (2) 93 (1971), 439-458. MR 43 \# 7668.

9. _ Function theory of finite order on algebraic varieties. I, II, J. Differential Geometry 6 (1972), 285-306; ibid. 7 (1972), 45-66. MR 46 \#3829; 48 \# 4345.

10. Some remarks on Nevanlinna theory, Value Distrubution Theory, Part A (Proc.

Tulane Univ. Program on Value-Distribution Theory in Complex Analysis and Related Topics in Differential Geometry, 1972-1973), Dekker, New York, 1974, pp. 1-11. MR 50 \# 5032.

11. Entire holomorphic mappings in one and several complex variables, Ann. of Math. Studies no. 85, Princeton Univ. Press, Princeton, N.J., 1976, pp. 1-99.

12. $\mathrm{Ph}$. Griffiths and $\mathrm{J}$. King, Nevanlinna theory and holomorphic mappings between algebraic varieties, Acta. Math. 130 (1973), 145-220.

13. J. Hirschfelder, The first main theorem of value distribution in several variables, Invent. Math. 8 (1969), 1-33. MR 39 \# 7146.

14. , On Wu's form of the first main theorem of value distribution in several variables, Proc. Amer. Math. Soc. 23 (1969), 548-554. MR 40 \#861.

15. H. Kneser, Zur Theorie der gebrochenen Funktionen mehrerer Veränderlichen, Jber. Deutsch. Math.-Verein 48 (1938), 1-28.

16. P. Lelong, Fonctiones entières (n-variables) et fonctions plurisousharmoniques d'ordre fini dans $\mathbf{C}^{n}$, J. Analyse Math. 12 (1964), 365-407. MR 29 \#3668.

17. H. Levine, $A$ theorem on holomorphic mappings into complex projective space, Ann. of Math. (2) 71 (1960), 529-535. MR 22 \#8531.

18. Y. Matsushima, On a problem of Stoll concerning a cohomology map from a flag manifold into a Grassmann manifold, Osaka J. Math. (to appear).

19. J. Murray, $A$ second main theorem of value distribution theory on Stein manifolds with pseudoconvex exhaustion, Thesis, Notre Dame, 1974, pp. 1-69.

20. R. Nevanlinna, Eindeutige analytische Funktionen, 2nd. ed., Die Grundlehren der math. Wissenschaften, Band 46, Springer-Verlag, Berlin, 1953, pp. 1-379. MR 15, 208.

21. W. Stoll, Die beiden Hauptsätze der Wertverteilungstheorie bie Funktionen mehrerer komp- 
lexer Veränderlichen. I, II, Acta Math. 90 (1953), 1-115; ibid. 92 (1954), 55-169. MR 17 \#893, 894.

22. , A general first main theorem of value distribution, Acta Math. 118 (1967), 111191. MR $36 \# 430$ a, b.

23, , About the value distribution of holomorphic maps into projective space, Acta Math. 123 (1969), 83-114. MR 41 \#3815.

24. Notes in Math., vol. 135, Springer-Verlag, Berlin and New York, 1970,pp. 1-267. MR 42 \#2040.

25. , Value distribution of holomorphic maps, Several Complex Variables. I (Proc. Conf., Univ. of Maryland, College Park, Md., 1970), Springer-Verlag, Berlin and New York, 1970, pp. 165-190. MR 42 \#4774.

26. , Deficit and Bézout estimates, Value-Distribution Theory, Part B (R. A. Kujala and A. L. Vitter III, Editors), Pure and Appl. Math, no 25, Dekker, New York, 1973, pp. 1273 MR 49 \# 9248.

27. _ Value distribution theory on parabolic spaces, Lecture, Notre Dame (to appear).

28., A Casorati Weierstrass theorem for Schubert zeroes of semi-ample holomorphic vector bundles, Memorie dell'Accademia Nazionale dei Lincei. (to appear).

29. Ch. Tung, The first main theorem on complex spaces, Thesis, Notre Dame, 1973, pp. 1-320.

30. H. Weyl and J. Weyl, Meromorphic functions and analytic curves, Ann. of Math. Studies, no. 12, Princeton Univ. Press, Princeton, N.J., 1943, pp. 1-269. MR 5, 94.

31. P. Wong, Defect relations for meromorphic maps from parabolic manifolds into complex projective spaces, Thesis, Notre Dame, 1976, pp. 1-231.

32. $\mathrm{H}$. Wu, Remarks on the first main theorem in equidistribution thoery. I, II, III, IV, J. Differential Geometry 2 (1968), 197-202; 369-384; ibid. 3 (1969), 83-94; 433-446. MR 43 \#2247a, b, c, d.

33. - The equidistribution theory of holomorphic curves, Ann. of Math. Studies, no. 64, Princeton Univ. Press, Princeton, N.J.; Univ. of Tokyo Press, Tokyo, 1970, pp. 1-219. MR 42 \#7951.

Department of Mathematics, University of Notre Dame, Notre Dame, Indiana 46556 